IMA Journal of Numerical Analysis (2010) 30, 870-885

doi:10.1093/imanum/drn081

Advance Access publication on April 7, 2009

\title{
Interior-penalty-stabilized Lagrange multiplier methods for the finite-element solution of elliptic interface problems
}

\author{
ERIK BURMAN \\ Institute of Analysis and Scientific Computing, Ecole Polytechnique Federale de Lausanne, \\ Station 8 CH-1015 Lausanne, Switzerland \\ AND \\ Peter Hansbo $\dagger$ \\ Department of Mathematical Sciences, Chalmers University of Technology and Göteborg \\ University, S-412 96 Göteborg, Sweden
}

[Received on 12 June 2008; revised on 4 November 2008]

\begin{abstract}
In this paper we propose a class of jump-stabilized Lagrange multiplier methods for the finite-element solution of multidomain elliptic partial differential equations using piecewise-constant or continuous piecewise-linear approximations of the multipliers. For the purpose of stabilization we use the jumps in derivatives of the multipliers or, for piecewise constants, the jump in the multipliers themselves, across element borders. The ideas are illustrated using Poisson's equation as a model, and the proposed method is shown to be stable and optimally convergent. Numerical experiments demonstrating the theoretical results are also presented.
\end{abstract}

Keywords: interface problem; non-matching grids; edge stabilization.

\section{Introduction}

Patching together possibly unrelated meshes across an interface (artificial or real) using Lagrange multiplier techniques requires that the relation between the discrete spaces chosen for the primal variable and the multipliers is such that the resulting numerical scheme is stable. Proving stability reduces to proving that the approximate solution fulfils the inf-sup condition (cf. Brezzi \& Fortin, 1991), which strongly restricts the possible choices of balance between the multiplier and the primal variable. One way around this problem is to use stabilized multiplier methods as in Barbosa \& Hughes (1992), Becker et al. (2003) and Hansbo et al. (2005). These are typically of least squares type, meaning that the stability is obtained via a least squares control of the residual of the multiplier equation. In this paper we suggest, instead, a stabilization scheme more in the vein of Burman \& Hansbo (2004), i.e., based on jumps in derivatives of the multiplier (or the multiplier itself) across element edges.

Another practical problem is the implementation of integration of products of traces of the primal variable and the multiplier. Stable methods (see Wohlmuth, 2001) typically use one of the trace meshes for the multipliers, and most stabilized methods use the jump in the primal variable as a part of the stabilization (for an exception see Hansbo et al., 2005). This means that piecewise polynomials on unrelated, unstructured meshes have to be integrated. By using stabilization solely involving the multiplier itself,

†Corresponding author. Email: hansbo@am.chalmers.se

(c) The author 2009. Published by Oxford University Press on behalf of the Institute of Mathematics and its Applications. All rights reserved. 
it is possible to use a third, for example, completely structured, mesh for the multiplier, which may help considerably in the integration problem.

An outline of the paper is as follows. In Section 2 we introduce our model problem, together with some notation, and present the interface Lagrange multiplier method with a generic discretization of the multiplier. The stability and error analysis of the new method are carried out in Section 3, and numerical experiments demonstrating the theoretical results are presented in Section 4.

\section{Formulation of the method}

In this section we introduce an interface Lagrange multiplier method for the finite-element discretization of elliptic problems on non-matching grids. Before doing that, we make precise the model problem that we will be working on, together with some notation and motivation of the present work.

\subsection{Model problem}

Let $\Omega$ be a bounded domain in $\mathbb{R}^{n}$, where $n=2$ or 3 , with boundary $\partial \Omega$. As a model problem, we consider a stationary heat conduction problem in the case where there is a piecewise straight internal boundary $\Gamma$ dividing $\Omega$ into two subdomains $\Omega_{1}$ and $\Omega_{2}$. Thus we want to solve for $u$ the problem

$$
\begin{aligned}
-\Delta u_{i}=f & \text { in } \Omega_{i}, \\
u_{i}=0 & \text { on } \partial \Omega_{i} \cap \partial \Omega, \\
u_{1}-u_{2}=0 & \text { on } \Gamma, \\
\boldsymbol{n}_{1} \cdot \nabla u_{1}+\boldsymbol{n}_{2} \cdot \nabla u_{2}=0 & \text { on } \Gamma
\end{aligned}
$$

for $i=1,2$, where we have denoted by $u_{i}$ the restriction of $u$ to $\Omega_{i}$. Here $f$ is a given function, $\Delta$ denotes the Laplace operator and $\boldsymbol{n}_{i}$ is the outward pointing normal to $\Omega_{i}$ at $\Gamma$, where $i=1,2$. We assume that the interface $\Gamma$ is decomposed as the union $\Gamma=\overline{\bigcup \Gamma_{j}}$ of $n_{\Gamma}$ straight lines (planes) $\Gamma_{j}$ of size $\ell_{j}$. We remark that two different situations can occur from a geometric point of view (see Fig. 1):

1. both $\partial \Omega_{1} \cap \partial \Omega$ and $\partial \Omega_{2} \cap \partial \Omega$ have nonzero $(n-1)$-dimensional measure;

2. either $\partial \Omega_{1} \cap \partial \Omega$ or $\partial \Omega_{2} \cap \partial \Omega$ has zero $(n-1)$-dimensional measure.

Define

$$
V=\left\{v: v_{i} \in H^{1}\left(\Omega_{i}\right), v_{i}=0 \text { on } \partial \Omega_{i} \backslash \Gamma, i=1,2\right\}
$$

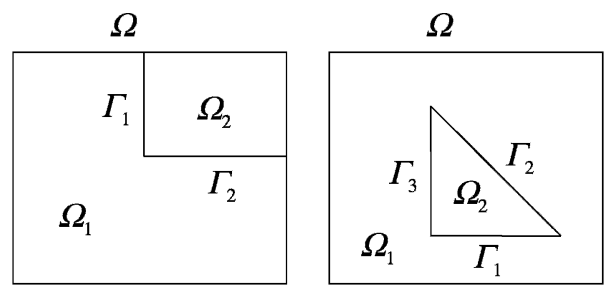

FIG. 1. Intersection and nonintersection of $\Gamma$ and $\partial \Omega$ in the case $\Omega \subset \mathbb{R}^{2}$. 
and $\Lambda=H^{-1 / 2}(\Gamma)$. A weak form of (2.1) using the Lagrange multiplier approach is then as follows. Find $(u, \lambda) \in V \times \Lambda$ such that

$$
\begin{aligned}
& \sum_{i} \int_{\Omega_{i}} \nabla u_{i} \cdot \nabla v_{i} \mathrm{~d} x+\int_{\Gamma} \lambda \llbracket v \rrbracket \mathrm{d} s=\sum_{i} \int_{\Omega_{i}} f v_{i} \mathrm{~d} x \quad \forall v \in V, \\
& \int_{\Gamma} \llbracket u \rrbracket \mu \mathrm{d} s=0 \quad \forall \mu \in \Lambda,
\end{aligned}
$$

where $\llbracket v \rrbracket:=\left.\left(v_{1}-v_{2}\right)\right|_{\Gamma}$ is the jump of $v$ across $\Gamma$. Note that, formally, we have

$$
\lambda=-\boldsymbol{n}_{1} \cdot \nabla u_{1}=\boldsymbol{n}_{2} \cdot \nabla u_{2} \quad \text { on } \Gamma \text {. }
$$

\subsection{Notation}

We introduce the necessary notation for the definition of the method that we are going to present and its subsequent analysis, focusing, for simplicity, on the case of tetrahedral elements. Therefore we assume that we are given a tetrahedral mesh $\mathscr{T}_{i}^{h}$ of the domain $\Omega_{i}$, where $i=1,2$. We denote by $h_{i}$ the mesh size of $\mathscr{T}_{i}^{h}$. Obviously, $\mathscr{T}^{h}=\mathscr{T}_{1}^{h} \cup \mathscr{T}_{2}^{h}$ provides a mesh for $\Omega$, whose mesh size is $h=\max \left\{h_{1}, h_{2}\right\}$. We introduce the (family of) finite-element spaces

$$
V^{h}=\left\{v^{h} \in V:\left.v^{h}\right|_{K} \in P^{1}(T) \quad \forall T \in \mathscr{T}^{h}\right\},
$$

where $P^{1}(T)$ denotes the space of linear polynomials on $T$.

We assume that each $\Gamma_{j}$ has been triangulated into a mesh $\mathscr{G}_{j}^{h}$ of simplices $K$ with size $h_{\Gamma_{j}}$. We use the notation $h_{\Gamma}$ for the function such that $\left.h_{\Gamma}\right|_{\Gamma_{j}}=h_{\Gamma_{j}}$. We assume that the trace meshes $\partial \mathscr{T}_{i, j}^{h}$ on $\Gamma_{j}$ and the multiplier mesh $\mathscr{G}_{j}^{h}$ are all shape regular. Further, viewing the mesh size parameters as piecewise-constant functions on the respective meshes, we assume that there holds a local quasiuniformity for the trace meshes in the sense that there are global constants $c_{1}$ and $c_{2}$ such that, for each $x \in \Gamma$, we have $c_{1} h_{i, j}(x) \leqslant h_{\Gamma, j} \leqslant c_{2} h_{i, j}(x)$ for $i=1,2$, where $h_{i, j}$ is the mesh size parameter of $\partial \mathscr{T}_{i, j}^{h}$. In the analysis $C$ will denote a generic constant that is independent of the mesh size, but not necessarily of the constants $c_{1}$ and $c_{2}$ or the local mesh geometry. We now introduce the space for the approximation of the Lagrange multipliers as

$$
\Lambda^{h}:=\left\{\mu^{h}:\left.\mu^{h}\right|_{K} \in P^{l}(K) \quad \forall K \in \mathscr{G}_{j}^{h}, j=1, \ldots, n_{\Gamma}\right\},
$$

with $l=0$ or $l=1$, and for $l=1$ we let $\mu^{h}$ be globally continuous on each $\Gamma_{j}$.

\subsection{Interior penalty stabilization}

We propose the following method.

Find $\left(u^{h}, \lambda^{h}\right) \in V^{h} \times \Lambda^{h}$ such that

$$
\begin{aligned}
& \sum_{i} \int_{\Omega_{i}} \nabla u_{i}^{h} \cdot \nabla v_{i}^{h} \mathrm{~d} x+\int_{\Gamma} \lambda^{h} \llbracket v^{h} \rrbracket \mathrm{d} s=\sum_{i} \int_{\Omega_{i}} f v_{i}^{h} \mathrm{~d} x \quad \forall v^{h} \in V^{h}, \\
& \int_{\Gamma} \llbracket u^{h} \rrbracket \mu^{h} \mathrm{~d} s-j\left(\lambda^{h}, \mu^{h}\right)=0 \quad \forall \mu^{h} \in \Lambda^{h},
\end{aligned}
$$


where

$$
\begin{aligned}
j(\lambda, \mu) & :=\sum_{j=1}^{n_{\Gamma}} \sum_{K \in \mathscr{G}_{j}^{h}} \int_{\partial K} \gamma h_{\partial K}^{2}[\lambda][\mu] \mathrm{d} s \quad \text { if } l=0, \\
j(\lambda, \mu) & :=\sum_{j=1}^{n_{\Gamma}} \sum_{K \in \mathscr{G}_{j}^{h}} \int_{\partial K} \gamma h_{\partial K}^{4}\left[\nabla_{\Gamma} \lambda\right] \cdot\left[\nabla_{\Gamma} \mu\right] \mathrm{d} s \quad \text { if } l=1 .
\end{aligned}
$$

Here $h_{\partial K}$ is the mean size of the elements sharing $\partial K,[q]$ is the jump of $q$ across $\partial K$ for $\partial K \cap \partial \Gamma_{i}=\emptyset$, $[q]=0$ on $\partial K \cap \partial \Gamma_{i}$ for $l=0,\left[\nabla_{\Gamma} q\right]=\nabla_{\Gamma} q$ for $l=1$ on $\partial K \cap \partial \Gamma_{i}$, and $\gamma$ is a constant. By $\nabla_{\Gamma}$ we denote the gradient in the plane of $\Gamma$.

REMARK 2.1 Another possible choice of stabilization operator is

$$
j(\lambda, \mu):=\sum_{j=1}^{n_{\Gamma}} \sum_{K \in \mathscr{G}_{j}^{h}} \int_{K} \gamma h_{\partial K}^{3} \nabla_{\Gamma} \lambda \nabla_{\Gamma} \mu \mathrm{d} x \quad \text { if } l=1 .
$$

The analysis of this method is included as a special case of the one given below. Details are left to the reader.

We note that (2.4) is a weakly consistent method: inserting a sufficiently regular analytical solution $(u, \lambda)$ in the place of $\left(u^{h}, \lambda^{h}\right)$, we find that

$$
\begin{gathered}
\sum_{i} \int_{\Omega_{i}} \nabla\left(u_{i}-u_{i}^{h}\right) \cdot \nabla v_{i}^{h} \mathrm{~d} x+\int_{\Gamma}\left(\lambda-\lambda^{h}\right) \llbracket v^{h} \rrbracket \mathrm{d} s=0, \\
\int_{\Gamma} \llbracket u-u^{h} \rrbracket \mu^{h} \mathrm{~d} s=-j\left(\lambda^{h}, \mu^{h}\right)
\end{gathered}
$$

for all $v^{h} \in V^{h}$ and $\mu^{h} \in \Lambda^{h}$. We rephrase this property in abstract form in the following lemma, where we set

$$
\mathscr{B}^{h}(u, \lambda ; v, \mu):=\sum_{i} \int_{\Omega_{i}} \nabla u_{i} \cdot \nabla v_{i} \mathrm{~d} x+\int_{\Gamma} \lambda \llbracket v \rrbracket \mathrm{d} s-\int_{\Gamma} \llbracket u \rrbracket \mu \mathrm{d} s .
$$

LEMMA 2.2 The method (2.4) is weakly consistent in the sense that

$$
\mathscr{B}^{h}\left(u-u^{h}, \lambda-\lambda^{h} ; v^{h}, \mu^{h}\right)=-j\left(\lambda^{h}, \mu^{h}\right)
$$

for all $v^{h} \in V^{h}$ and $\mu^{h} \in \Lambda^{h}$.

\section{Analysis of the method}

For the analysis we introduce the triple norm (defined on $V \times L_{2}(\Gamma)$ for $\xi=0$, and For $\xi=1$ the norm is used on functions in the discrete spaces $V^{h}$ and $\Lambda^{h}$ ) as

$$
\|(v, \mu)\|_{\xi}^{2}:=\|\nabla v\|_{0, h}^{2}+\|\mu\|_{-\frac{1}{2}, h, \Gamma}^{2}+\xi j(\mu, \mu),
$$


where

$$
\|v\|_{0, h}^{2}:=\sum_{i}\|v\|_{L_{2}\left(\Omega_{i}\right)}^{2}, \quad\|\mu\|_{-\frac{1}{2}, h, \Gamma}^{2}:=\int_{\Gamma} h_{\Gamma} \mu^{2} \mathrm{~d} s .
$$

We will also use the discrete half-norm

$$
\|v\|_{\frac{1}{2}, h, \Gamma}^{2}:=\int_{\Gamma} h_{\Gamma}^{-1} v^{2} \mathrm{~d} s
$$

and note for future reference that

$$
\int_{\Gamma} \mu \llbracket v \rrbracket \mathrm{d} s \leqslant\|\mu\|_{-\frac{1}{2}, h, \Gamma}\|\llbracket v \rrbracket\|_{\frac{1}{2}, h, \Gamma} .
$$

REMARK 3.1 We note that, in general we have coercivity of $\mathscr{B}^{h}(u, 0 ; v, 0)$ on $Y$, where

$$
Y=\left\{v \in H^{1}\left(\Omega_{1}\right) \times H^{1}\left(\Omega_{2}\right):\left.v\right|_{\partial \Omega}=0, \int_{\Gamma} \llbracket v \rrbracket \mathrm{d} s=0\right\}
$$

(cf. Wohlmuth, 2001). By choosing $\mu=1$ in (2.2) we have

$$
\int_{\Gamma} \llbracket u \rrbracket \mathrm{d} s=0,
$$

and hence we can look for a solution only in the subspace $V_{h} \cap Y$ on which the coercivity holds. In the case when both $\partial \Omega_{1} \cap \partial \Omega$ and $\partial \Omega_{2} \cap \partial \Omega$ have nonzero $(n-1)$-dimensional measure, coercivity instead follows directly from a standard Poincaré inequality. In that case we, however, have to use

$$
\Lambda=\left(H_{00}^{1 / 2}(\Gamma)\right)^{\prime}
$$

the dual space of $H_{00}^{1 / 2}(\Gamma)$ (for a formal definition of this space see, e.g., Lions \& Magenes, 1968).

We next define a quasi-interpolation operator $\pi_{i}$ as the standard nodal interpolation operator onto the trace mesh $\partial \mathscr{T}_{i, j}^{h}$ on $\Gamma_{j}$ of the mesh on $\Omega_{i}$ in the case of a continuous multiplier space. In the case of a discontinuous multiplier space, $\pi_{i}$ is defined by

$$
\pi_{i} \lambda^{h}\left(x_{k}\right)=\left.\frac{1}{n_{k}} \sum_{\left\{\tilde{K} \in \mathscr{G}_{j}^{h}: x_{k} \in \tilde{K}\right\}} \lambda^{h}\left(x_{k}\right)\right|_{\tilde{K}},
$$

where $x_{k}$ denotes the coordinate of node number $k$ in the trace mesh and $n_{k}$ denotes the cardinality of the set of elements $\left\{\tilde{K} \in \mathscr{G}_{j}^{h}: x_{k} \in \tilde{K}\right\}$. The constant $C_{\lambda}$ depends on the quasi-uniformity constants $c_{1}$ and $c_{2}$. A cornerstone in the analysis of the edge-stabilized Lagrange multiplier method is then the following discrete interpolation lemma.

LEMMA 3.2 For $\lambda^{h} \in \Lambda^{h}$ we have

$$
\left\|\lambda^{h}-\pi_{i} \lambda^{h}\right\|_{-\frac{1}{2}, h, \Gamma}^{2} \leqslant C_{j} j\left(\lambda^{h}, \lambda^{h}\right) .
$$


Proof. We first consider the case of piecewise-constant multipliers. Write $\left\|\lambda^{h}-\pi_{i} \lambda^{h}\right\|_{-\frac{1}{2}, h, \Gamma}^{2}$ as the sum over the triangles of the trace meshes $\partial \mathscr{T}_{i, j}^{h}$ as follows:

$$
\left\|\lambda^{h}-\pi_{i} \lambda^{h}\right\|_{-\frac{1}{2}, h, \Gamma}^{2}=\sum_{j=1}^{n_{\Gamma}} \sum_{K \in \partial \mathscr{T}_{i, j}^{h}}\left\|h_{\Gamma}^{1 / 2}\left(\lambda^{h}-\pi_{i} \lambda^{h}\right)\right\|_{L_{2}(K)}^{2} .
$$

Let $\tilde{\mathscr{K}}$ be the set of all of the triangles $\tilde{K}$ in $\mathscr{G}_{j}^{h}$ such that the measure of $K \cap \tilde{K}$ is nonzero and let $\tilde{\mathscr{F}}$ be the set of all faces $\tilde{F} \in \mathscr{G}_{j}^{h}$ such that the measure of $K \cap \tilde{F}$ is nonzero. Let $\tilde{V}$ denote the space of functions $e_{\pi}=\lambda^{h}-\pi_{i} \lambda^{h}$ associated with $\tilde{\mathscr{K}}$.

We consider an affine map from the reference element $M(\hat{\mathbf{x}})=M_{K} \hat{\mathbf{x}}+\mathbf{b}_{K}$. This mapping is also used for the overlapped patch of elements (see Fig. 2). The proof now goes by norm equivalence on discrete spaces. We will prove that the jump operator is a norm of the space $\tilde{V}$ and then conclude by a scaling argument. By the shape regularity and the local quasi-uniformity assumption on the trace meshes, we know that the dimension of the space $\tilde{V}$ is bounded uniformly in $h$. Clearly, if $\left.\left[e_{\pi}\right]\right|_{\tilde{F}}=$ $\left.\left[\lambda^{h}\right]\right|_{\tilde{F}}=0$ for all $\tilde{F} \in \tilde{\mathscr{F}}$ then $\lambda^{h}$ is constant over $\tilde{\mathscr{K}}$, and hence $e_{\pi}$ is zero. As a consequence, the following inequality holds:

$$
\left\|\lambda^{h}-\pi_{i} \lambda^{h}\right\|_{L_{2}(K)}^{2}=\int_{\hat{K}}\left|\lambda^{h}-\pi_{i} \lambda^{h}\right|^{2}\left|\operatorname{det}\left(M_{K}\right)\right| \mathrm{d} \hat{x} \leqslant\left.\tilde{c} \sum_{\tilde{F} \in \tilde{\mathscr{F}}}\left[\lambda^{h}\right]\right|_{\tilde{F}} ^{2}\left|\operatorname{det}\left(M_{K}\right)\right|,
$$

where we have used that meas $(\hat{K})=1$ and $\tilde{c}$ is uniformly bounded since $\operatorname{dim}(\tilde{V})$ is bounded. Moreover, on the reference element we have

$$
\left.\tilde{c} \sum_{\tilde{F} \in \tilde{\mathscr{F}}}\left[\lambda^{h}\right]\right|_{\tilde{F}} ^{2}\left|\operatorname{det}\left(M_{K}\right)\right| \leqslant \tilde{c}_{1} \sum_{\tilde{F} \in \tilde{\mathscr{F}}} \int_{\tilde{F}}\left[\lambda^{h}\right]^{2}\left|\operatorname{det}\left(M_{K}\right)\right| \mathrm{d} \hat{s} .
$$
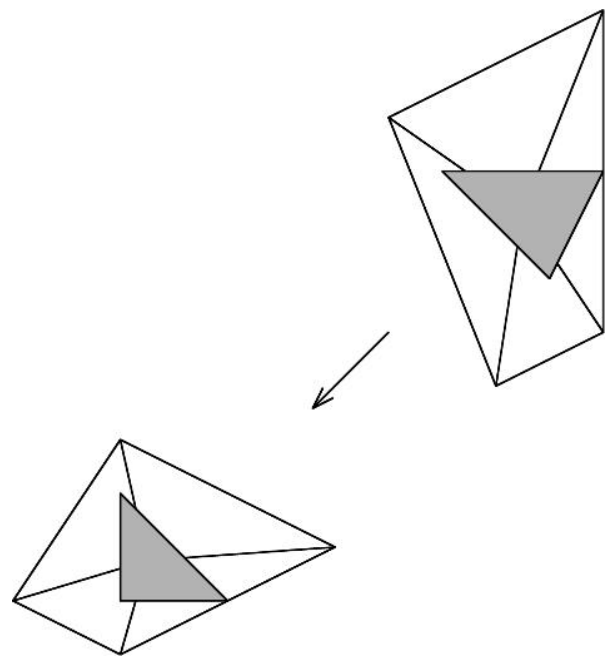

FIG. 2. Affine map $M$ from a reference element, marked grey. 
Scaling back to the physical element, we have

$$
\tilde{c}_{1} \sum_{\tilde{F} \in \tilde{\mathscr{F}}} \int_{\tilde{F}}\left[\lambda^{h}\right]^{2}\left|\operatorname{det}\left(M_{K}\right)\right| \mathrm{d} \hat{s} \leqslant \tilde{c}_{1} \sum_{\tilde{F} \in \tilde{\mathscr{F}}} \int_{\tilde{F}}\left[\lambda^{h}\right]^{2}\left|M_{K}\right| \mathrm{d} s,
$$

and, since $\left|M_{K}\right|=\rho_{\hat{K}}^{-1} h_{K}$ (where $\rho_{\hat{K}}$ is the radius of the largest inscribed disc in the reference element), we may conclude.

We finally consider the second case: continuous piecewise affine functions. The proof in this case is similar to the previous one, but simpler since $e_{\pi}$ must always be zero at the interpolation points. Assume, once again, that $j\left(e_{\pi}, e_{\pi}\right)=0$. This means that $e_{\pi} \in P^{1}$ globally on $\tilde{K}$, but it vanishes at the interpolation points and must therefore be zero since polynomials of order 1 are uniquely defined by the interpolation points on the reference element. Hence $j\left(e_{\pi}, e_{\pi}\right)$ is a norm on the space $\tilde{V}$. Once again, we may conclude by scaling and summation over the elements.

Lemma 3.2 is insufficient for stability due to the presence of corners or Dirichlet boundary conditions. The reason for this is that, to prove the inf-sup condition we wish to choose a function $v_{\lambda}^{h} \in V_{h}$, which is a harmonic extension in $\Omega_{1}$, such that

$$
\left.v_{\lambda}^{h}\right|_{\partial \Omega_{1} \cap \Gamma}=\pi_{1} h_{\Gamma} \lambda^{h}, \quad v_{\lambda}^{h}=0 \quad \text { in } \Omega_{2} .
$$

Unfortunately, if boundary conditions are imposed strongly then $u_{\lambda}^{h}$ must be zero in the part of $\Gamma$ intersecting the boundary to be a member of $V^{h}$, or if $\Gamma$ has corners then $\pi_{1} h_{\Gamma} \lambda^{h}$ will be double valued in the corner (in two space dimensions) and clearly the continuous $u_{\lambda}^{h}$ cannot fulfil the jump in $\pi_{1} h_{\Gamma} \lambda^{h}$. We will show how this problem can be solved by modifying the interpolant by simply setting it to zero at the problematic points. To keep down technical details we restrict ourselves to the two-dimensional case. We introduce the modified interpolant. Let $\partial \mathscr{G}_{j}^{h}$ denote the trace mesh of $\mathscr{G}_{j}^{h}$. Then

$$
\pi_{1,0} \lambda^{h}\left(x_{k}\right)= \begin{cases}0 & \text { if } x_{k} \in \partial K \subset \partial \mathscr{G}_{j}^{h}, \\ \pi_{1} \lambda^{h}\left(x_{k}\right) & \text { otherwise. }\end{cases}
$$

In the following important lemma we show that the missing portion in corners or on Dirichlet boundaries can be controlled by the stabilization operator as well.

LEMMA 3.3 If $\lambda^{h} \in \Lambda^{h}$ then we have

$$
\int_{\Gamma} \lambda^{h} \pi_{1,0} h_{\Gamma} \lambda^{h} \mathrm{~d} s \geqslant \frac{1}{6}\left\|\lambda^{h}\right\|_{-\frac{1}{2}, h, \Gamma}^{2}-C_{\lambda j} j\left(\lambda^{h}, \lambda^{h}\right) .
$$

Proof. It is sufficient to show the inequality on one of the sides $\Gamma_{j}$. First let $\Gamma_{E}=\left\{x \in \Gamma_{j}: \pi_{1,0} \lambda^{h} \neq\right.$ $\left.\pi_{1} \lambda^{h}\right\}$ and $\Gamma_{I}=\Gamma_{j} \backslash \Gamma_{E}$. We may then write

$$
\begin{aligned}
\int_{\Gamma_{j}} \lambda^{h} h_{\Gamma} \pi_{1,0} \lambda \mathrm{d} s & =\left\|\lambda^{h}\right\|_{-\frac{1}{2}, h, \Gamma_{I}}^{2}+\int_{\Gamma_{I}} \lambda^{h} h_{\Gamma}\left(\pi_{1} \lambda-\lambda^{h}\right) \mathrm{d} s+\int_{\Gamma_{E}} \lambda^{h} \pi_{1,0} \lambda \mathrm{d} s \\
& =\left\|\lambda^{h}\right\|_{-\frac{1}{2}, h, \Gamma_{I}}^{2}+I_{1}+I_{2} .
\end{aligned}
$$

We first consider the term $I_{1}$. It follows from the Cauchy-Schwarz inequality and Lemma 3.1 that

$$
I_{1} \geqslant-\frac{1}{2}\left\|\lambda^{h}\right\|_{-\frac{1}{2}, h, \Gamma_{j}}^{2}-C_{j} j\left(\lambda^{h}, \lambda^{h}\right) .
$$


The second part, however, requires a slightly more intricate analysis. Now $\Gamma_{E}$ consists of the extremal intervals of $\Gamma_{j}$ that we will denote by $\Gamma_{E, 0}$ and $\Gamma_{E, 1}$. We only consider one end interval. Let $\Gamma_{E, 0}$ be parameterized by $\left(0, x_{E}\right)$, where 0 is the end point of $\bar{\Gamma}_{j}$ and $x_{E}$ is the interior point such that $\bar{\Gamma}_{E, 0} \cap \Gamma_{I}=x_{E}$. We write $\lambda_{E}=\pi_{1} \lambda^{h}\left(x_{E}\right)$. It then follows that

$$
\begin{aligned}
I_{2} & =\int_{\Gamma_{E, 0}}\left(\lambda^{h}-\lambda_{E}\right) h_{\Gamma} \pi_{1,0} \lambda^{h} \mathrm{~d} s+\int_{\Gamma_{E, 0}} \lambda_{E} h_{\Gamma} \pi_{1,0} \lambda^{h} \mathrm{~d} s \\
& \geqslant-\frac{1}{2}\left\|\lambda_{E}-\lambda^{h}\right\|_{-\frac{1}{2}, h, \Gamma_{E, 0}}^{2}-\frac{1}{2}\left\|\pi_{1,0} \lambda^{h}\right\|_{-\frac{1}{2}, h, \Gamma_{E, 0}}^{2}+\int_{\Gamma_{E, 0}} \lambda_{E} h_{\Gamma} \pi_{1,0} \lambda^{h} \mathrm{~d} s .
\end{aligned}
$$

Note that $\left|\left(\lambda_{E}-\lambda^{h}\right)\left(x_{E}\right)\right| \leqslant \frac{1}{2}\left|\left[\lambda^{h}\left(x_{E}\right)\right]\right|$. It then follows by a discrete Poincaré inequality that $\| \lambda_{E}-$ $\lambda^{h} \|_{-\frac{1}{2}, h, \Gamma_{E}}^{2} \leqslant C j\left(\lambda^{h}, \lambda^{h}\right)$ in the case of piecewise-constant multiplicator spaces. For the case of the piecewise affine multiplicator space the nonconsistent part is also needed. Indeed, in the case of affine Lagrange multiplicators, since $\left|\left(\lambda_{E}-\lambda^{h}\right)\left(x_{E}\right)\right|=0$, a Poincaré inequality yields that

$$
\left\|\lambda_{E}-\lambda^{h}\right\|_{0, \Gamma_{E, 0}}^{2} \leqslant C\left\|h_{E} \nabla_{\Gamma} \lambda^{h}\right\|_{\Gamma_{E, 0}}^{2}
$$

where $h_{E}=x_{E} \leqslant c h_{\Gamma}$. The gradient of $\lambda^{h}$ cannot be controlled solely by the jumps since we do not have $\nabla_{\Gamma} \lambda^{h}=0$ somewhere in $\Gamma_{E, 0}$. However, since by definition $\left.\left[\nabla_{\Gamma} \lambda^{h}\right]\right|_{f}=\left.\nabla_{\Gamma} \lambda^{h}\right|_{f}$ for $f \in \partial \Gamma_{j}$, we have the Poincaré-type estimate

$$
\left\|\lambda_{E}-\lambda^{h}\right\|_{-\frac{1}{2}, h, \Gamma_{E, 0}}^{2} \leqslant C j\left(\lambda^{h}, \lambda^{h}\right)
$$

in this case as well.

The uniformity of the above bounds, of course, relies on the fact that the various trace meshes have 'similar' mesh sizes. If the mesh for the Lagrange multiplicators is strongly refined independent of the other trace meshes, then the constant $C$ above will become large.

Recalling that $\lambda_{E}$ is constant on $\Gamma_{E, 0}$ and $\pi_{1,0} \lambda^{h}=\left(x / x_{E}\right) \lambda_{E}$ on $\Gamma_{E, 0}$, one may easily evaluate the last two integrals of equation (3.5) to obtain

$$
\int_{\Gamma_{E, 0}} \lambda_{E} h_{\Gamma} \pi_{1,0} \lambda^{h} \mathrm{~d} s=\int_{\Gamma_{E, 0}} \lambda_{E} h_{\Gamma}\left(\frac{x}{x_{E}}\right) \lambda_{E} \mathrm{~d} s=\frac{1}{2}\left\|\lambda_{E}\right\|_{-\frac{1}{2}, h, \Gamma_{E, 0}}^{2}
$$

and

$$
\left\|\pi_{1,0} \lambda^{h}\right\|_{-\frac{1}{2}, h, \Gamma_{E, 0}}^{2}=\int_{\Gamma_{E, 0}} h_{\Gamma}\left(\pi_{1,0} \lambda^{h}\right)^{2} \mathrm{~d} s=\int_{\Gamma_{E, 0}} h_{\Gamma}\left(\frac{x}{x_{E}}\right)^{2} \lambda_{E}^{2} \mathrm{~d} s=\frac{1}{3}\left\|\lambda_{E}\right\|_{-\frac{1}{2}, h, \Gamma_{E, 0}}^{2} .
$$

Collecting the inequalities (3.3)-(3.5), we have

$$
\int_{\Gamma_{j}} \lambda^{h} h_{\Gamma} \pi_{1,0} \lambda^{h} \mathrm{~d} s \geqslant \frac{1}{2}\left\|\lambda^{h}\right\|_{-\frac{1}{2}, h, \Gamma_{I}}^{2}-C j\left(\lambda^{h}, \lambda^{h}\right)+\frac{1}{3} \sum_{i=0}^{1}\left\|\lambda_{E}\right\|_{-\frac{1}{2}, h, \Gamma_{E, i}}^{2}
$$

We may conclude using that, for $i=0,1$ we have

$$
\left\|\lambda^{h}\right\|_{-\frac{1}{2}, h, \Gamma_{E, i}}^{2} \leqslant 2\left\|\lambda_{E}\right\|_{-\frac{1}{2}, h, \Gamma_{E, i}}^{2}+2\left\|\lambda_{E}-\lambda^{h}\right\|_{-\frac{1}{2}, h, \Gamma_{E, i}}^{2} \leqslant 2\left\|\lambda_{E}\right\|_{-\frac{1}{2}, h, \Gamma_{E, i}}^{2}+C j\left(\lambda^{h}, \lambda^{h}\right),
$$


leading to the desired inequality

$$
\int_{\Gamma_{j}} \lambda^{h} h_{\Gamma} \pi_{1,0} \lambda^{h} \mathrm{~d} s \geqslant \frac{1}{2}\left\|\lambda^{h}\right\|_{-\frac{1}{2}, h, \Gamma_{I}}^{2}-C j\left(\lambda^{h}, \lambda^{h}\right)+\frac{1}{6} \sum_{i=0}^{1}\left\|\lambda^{h}\right\|_{-\frac{1}{2}, h, \Gamma_{E, i}}^{2} .
$$

LEMma 3.4 (Stability) Let $W^{h}=V^{h} \cap Y$, where $Y$ is defined in Remark 3.1. For all $\left(u^{h}, \lambda^{h}\right) \in W^{h} \times \Lambda^{h}$ we then have

$$
C\left\|\left(u^{h}, \lambda^{h}\right)\right\|_{1} \leqslant \sup _{\left(v^{h}, \mu^{h}\right) \in W^{h} \times \Lambda^{h}} \frac{\mathscr{B}^{h}\left(u^{h}, \lambda^{h} ; v^{h}, \mu^{h}\right)+j\left(\lambda^{h}, \mu^{h}\right)}{\left\|\left(v^{h}, \mu^{h}\right)\right\|_{1}} .
$$

Proof. Assume that $\left(u^{h}, \lambda^{h}\right) \in W^{h} \times \Lambda^{h}$. Consider the harmonic extension $u_{\lambda}^{h} \in V_{h}$ such that

$$
\left.u_{\lambda}^{h}\right|_{\partial \Omega_{1} \cap \Gamma}=h_{\Gamma} \pi_{1,0} \lambda^{h}, \quad u_{\lambda}^{h}=0 \quad \text { in } \Omega_{2},
$$

for which, by equivalence on norms on discrete spaces and scaling, we have

$$
\left\|u_{\lambda}^{h}\right\|_{1, h} \leqslant c\left\|h_{\Gamma} \pi_{1,0} \lambda^{h}\right\|_{\frac{1}{2}, \Gamma} \leqslant C\left\|\pi_{1,0} \lambda^{h}\right\|_{-\frac{1}{2}, h, \Gamma} \leqslant C_{1}\left\|\lambda^{h}\right\|_{-\frac{1}{2}, h, \Gamma} .
$$

Take $\mu^{h}=\lambda^{h}$ and $v^{h}=v_{1}^{h}+\delta v_{2}^{h}$, where $v_{1}^{h}:=u^{h}, v_{2}^{h}:=u_{\lambda}^{h}$ and $\delta$ is a positive parameter to be chosen. We first note that, by (3.6), we have

$$
\left\|u_{\lambda}^{h}+u^{h}\right\|_{1, h} \leqslant C\left(\left\|\lambda^{h}\right\|_{-\frac{1}{2}, h, \Gamma}+\left\|u^{h}\right\|_{1, h}\right)
$$

and thus the continuity result

$$
\left\|\left(v^{h}, \mu^{h}\right)\right\| \leqslant C\left\|\left(u^{h}, \lambda^{h}\right)\right\|
$$

follows. Next we note that, by definition, we have

$$
\mathscr{B}^{h}\left(u^{h}, \lambda^{h} ; v_{1}^{h}, \mu^{h}\right)=\left\|u^{h}\right\|_{1, h}^{2}+j\left(\lambda^{h}, \lambda^{h}\right)
$$

and by applying Lemma 3.3 we obtain

$$
\begin{aligned}
B^{h}\left(u^{h}, \lambda^{h} ; v_{2}^{h}, 0\right) & =\int_{\Omega_{1}} \nabla u^{h} \cdot \nabla u_{\lambda}^{h} \mathrm{~d} x+\int_{\Gamma} \lambda^{h} h_{\Gamma} \pi_{1,0} \lambda^{h} \mathrm{~d} s \\
& \geqslant-\left\|u^{h}\right\|_{1, h}\left\|u_{\lambda}^{h}\right\|_{1, h}+\frac{1}{6}\left\|\lambda^{h}\right\|_{-\frac{1}{2}, h, \Gamma}^{2}-C_{\lambda} j\left(\lambda^{h}, \lambda^{h}\right) \\
& \geqslant-\frac{1}{2 \epsilon}\left\|u^{h}\right\|_{1, h}^{2}-\frac{\epsilon}{2}\left\|u_{\lambda}^{h}\right\|_{1, h}^{2}+\frac{1}{6}\left\|\lambda^{h}\right\|_{-\frac{1}{2}, h, \Gamma}^{2}-C_{\lambda} j\left(\lambda^{h}, \lambda^{h}\right) \\
& \geqslant-\frac{1}{2 \epsilon}\left\|u^{h}\right\|_{1, h}^{2}+\left(\frac{1}{6}-\frac{\epsilon}{2} C_{1}\right)\left\|\lambda^{h}\right\|_{-\frac{1}{2}, h, \Gamma}^{2}-C_{\lambda} j\left(\lambda^{h}, \lambda^{h}\right),
\end{aligned}
$$

where $\epsilon$ is at our disposal. Adding (3.8) and (3.9), choosing $\epsilon<1 /\left(12 C_{1}\right)$ and $\delta<\min \left(\epsilon, 1 /\left(2 C_{\lambda}\right)\right)$, followed by invoking (3.7), the statement of the lemma follows. 
Lemma 3.5 (Continuity) Provided that the multipliers are regular enough for the triple norm to make sense, for all $u \in V+V^{h}, \lambda \in \Lambda+\Lambda^{h}, v \in V^{h}$ and $\mu \in \Lambda^{h}$, we have

$$
\mathscr{B}^{h}(u, \lambda ; v, \mu) \leqslant C\left(\|(u, \lambda)\|_{0}+\|\lambda\|_{-\frac{1}{2}, \Gamma}+\|\llbracket u \rrbracket\|_{\frac{1}{2}, h, \Gamma}\right)\|(v, \mu)\| .
$$

Proof. The continuity follows immediately by the Cauchy-Schwarz inequality, the duality inequality

$$
\int_{\Gamma} \lambda \llbracket v \rrbracket \mathrm{d} s \leqslant\|\lambda\|_{-\frac{1}{2}, \Gamma}\|\llbracket v \rrbracket\|_{\frac{1}{2}, \Gamma}
$$

and its discrete counterpart (3.1), and by noting that, by a trace inequality, we have

$$
\|\llbracket v \rrbracket\|_{\frac{1}{2}, \Gamma} \leqslant\left\|v_{1}\right\|_{\frac{1}{2}, \Gamma}+\left\|v_{2}\right\|_{\frac{1}{2}, \Gamma} \leqslant C\|\nabla v\|_{0, h} .
$$

LEMMA 3.6 (Best approximation) We have

$$
\begin{array}{r}
\left\|\left(u-u^{h}, \lambda-\lambda^{h}\right)\right\|_{0} \leqslant C \inf _{\left(v^{h}, \mu^{h}\right) \in V^{h} \times \Lambda^{h}}\left(\|\|\left(u-v^{h}, \lambda-\mu^{h}\right)\|\|_{0}+\left\|\lambda-\mu^{h}\right\|_{-\frac{1}{2}, \Gamma}\right. \\
\left.+\left\|\llbracket u-v^{h} \rrbracket\right\|_{\frac{1}{2}, h, \Gamma}+j\left(\mu^{h}, \mu^{h}\right)^{1 / 2}\right) .
\end{array}
$$

Proof. Take $\left(v^{h}, \mu^{h}\right) \in V^{h} \times \Lambda^{h}$. By the triangle inequality we have

$$
\left\|\left(u-u^{h}, \lambda-\lambda^{h}\right)\right\| \leqslant\left\|\left(u-v^{h}, \lambda-\mu^{h}\right)\right\|_{0}+\left\|\left(v^{h}-u^{h}, \mu^{h}-\lambda^{h}\right)\right\|_{1},
$$

and by Lemmas 2.2, 3.4 and 3.5 we have that there exists $x^{h}$ and $y^{h}$ with $\left\|\left(x^{h}, y^{h}\right)\right\|_{1} \leqslant$ $\left\|\left(u^{h}-v^{h}, \lambda^{h}-\mu^{h}\right)\right\|_{1}$ such that

$$
\begin{aligned}
\left\|\left(u^{h}-v^{h}, \lambda^{h}-\mu^{h}\right)\right\|_{1}^{2} \leqslant & \leqslant \mathscr{B}^{h}\left(u^{h}-v^{h}, \lambda^{h}-\mu^{h} ; x^{h}, y^{h}\right)+j\left(\lambda^{h}-\mu^{h}, y^{h}\right) \\
& =C \mathscr{B}^{h}\left(u-v^{h}, \lambda-\mu^{h} ; x^{h}, y^{h}\right)-j\left(\mu^{h}, y^{h}\right) \\
& \leqslant C\left\|\left(u^{h}-v^{h}, \lambda^{h}-\mu^{h}\right)\right\|_{1}\left(\left\|\left(u-v^{h}, \lambda-\mu^{h}\right)\right\|_{0}+\left\|\lambda-\mu^{h}\right\|_{-\frac{1}{2}, \Gamma}\right. \\
& +\|\left[u-v^{h} \rrbracket \|_{\frac{1}{2}, h, \Gamma}+j\left(\mu^{h}, \mu^{h}\right)^{1 / 2}\right),
\end{aligned}
$$

which concludes the proof.

We now have the following a priori estimate.

THEOREM 3.7 (Convergence) With $u \in H^{2}(\Omega)$ and $\lambda \in H^{1 / 2}\left(\bigcup \Gamma_{j}\right)$, we have

$$
\left\|\left(u-u^{h}, \lambda-\lambda^{h}\right)\right\|_{0}+j\left(\lambda^{h}, \lambda^{h}\right) \leqslant C\left(h|u|_{H^{2}(\Omega)}+h_{\Gamma}|\lambda|_{H^{1 / 2}\left(\cup \Gamma_{j}\right)}\right) .
$$


Proof. In Lemma 3.6 choose $v^{h}=\pi_{h} u$ and $\mu^{h}=P_{l} \lambda$, where $\pi_{h}$ denotes the standard nodal interpolant in $V^{h}$, and $P_{0}$ and $P_{1}$ as the $L_{2}\left(\bigcup \Gamma_{j}\right)$-projection onto the piecewise-constant and piecewise-linear (continuous) spaces, respectively. Moreover, let $\pi_{h}^{1}$ and $\pi_{h}^{2}$ denote the different interpolants on the meshes on $\Omega_{1}$ and $\Omega_{2}$. Then, by standard estimates we have

$$
\begin{gathered}
\left\|\nabla\left(u-\pi_{h} u\right)\right\|_{0, h} \leqslant C h|u|_{H^{2}(\Omega)}, \\
\left\|\lambda-P_{l} \lambda\right\|_{-\frac{1}{2}, h, \Gamma_{j}} \leqslant C h_{\Gamma}|\lambda|_{H^{1 / 2}\left(\cup \Gamma_{j}\right)}, \quad l=0,1,
\end{gathered}
$$

and by interpolation between function spaces (cf. Hansbo et al., 2005) we have

$$
\left\|\lambda-P_{l} \lambda\right\|_{-\frac{1}{2}, \Gamma_{j}} \leqslant C h_{\Gamma}|\lambda|_{H^{1 / 2}\left(\cup \Gamma_{j}\right)}, \quad l=0,1 .
$$

Further,

$$
\left\|\llbracket u-\pi_{h} u \rrbracket\right\|_{\frac{1}{2}, h, \Gamma} \leqslant\left\|u-\pi_{h}^{1} u\right\|_{\frac{1}{2}, h, \Gamma}+\left\|u-\pi_{h}^{2} u\right\|_{\frac{1}{2}, h, \Gamma},
$$

and by the trace inequality we have

$$
\|v\|_{L_{2}(\partial K)} \leqslant C\left(h_{K}^{-1 / 2}\|v\|_{L_{2}(K)}+h_{K}^{1 / 2}\|\nabla v\|_{L_{2}(K)}\right)
$$

(cf. Thomée, 1997). We conclude that

$$
\left\|\llbracket u-\pi_{h} u \rrbracket\right\|_{\frac{1}{2}, h, \Gamma} \leqslant C h|u|_{H^{2}(\Omega)} .
$$

It remains to estimate the jump terms and the nonconsistent boundary term present for piecewise affine approximation of the multiplier. We have, for $l=0$, that

$$
\left\|h_{\partial K}\left[P_{0} \lambda\right]\right\|_{\partial K}=\left\|h_{\partial K}\left[P_{0} \lambda-P_{1} \lambda\right]\right\|_{\partial K},
$$

which we can split into contributions from the element $K$ and its neighbour, and by (3.17) we have, also using an inverse estimate, that

$$
\begin{aligned}
\left\|h_{\partial K}\left(P_{0} \lambda-P_{1} \lambda\right)\right\|_{\partial K} & \leqslant C\left(\left\|h_{K}^{1 / 2}\left(P_{0} \lambda-P_{1} \lambda\right)\right\|_{K}+\left\|h_{K}^{3 / 2} \nabla\left(P_{0} \lambda-P_{1} \lambda\right)\right\|_{K}\right) \\
& \leqslant C\left\|h_{K}^{1 / 2}\left(P_{0} \lambda-P_{1} \lambda\right)\right\|_{K} \\
& \leqslant C\left(\left\|h_{K}^{1 / 2}\left(P_{0} \lambda-\lambda\right)\right\|_{K}+\left\|h_{K}^{1 / 2}\left(\lambda-P_{1} \lambda\right)\right\|_{K}\right) .
\end{aligned}
$$

For $l=1$ we similarly have (for all $\partial K$, also those on $\partial \Gamma$ )

$$
\begin{aligned}
\left\|h_{\partial K}^{2} \nabla P_{1} \lambda\right\|_{\partial K} & \leqslant C\left\|h_{\Gamma}^{3 / 2} \nabla P_{1} \lambda\right\|_{K} \\
& =C\left\|h_{\Gamma}^{3 / 2} \nabla\left(P_{1} \lambda-P_{0} \lambda\right)\right\|_{K} \\
& \leqslant C\left\|h_{\Gamma}^{1 / 2}\left(P_{1} \lambda-P_{0} \lambda\right)\right\|_{K} \\
& \leqslant C\left(\left\|h_{K}^{1 / 2}\left(P_{1} \lambda-\lambda\right)\right\|_{K}+\left\|h_{K}^{1 / 2}\left(\lambda-P_{0} \lambda\right)\right\|_{K}\right) .
\end{aligned}
$$


Taking the sum over all of the elements, we find that

$$
j\left(P_{l} \lambda, P_{l} \lambda\right)^{1 / 2} \leqslant C h_{\Gamma}|\lambda|_{H^{1 / 2}\left(\cup \Gamma_{j}\right)}, \quad l=0,1,
$$

and by the triangle inequality we have

$$
j\left(\lambda^{h}, \lambda^{h}\right)^{1 / 2} \leqslant j\left(\lambda^{h}-P_{l} \lambda, \lambda^{h}-P_{l} \lambda\right)^{1 / 2}+j\left(P_{l} \lambda, P_{l} \lambda\right) .
$$

The above interpolation estimates combined with Lemma 3.6 (including the inequality (3.12)) concludes the proof.

Finally, we give a second-order convergence estimate in $L_{2}(\Omega)$ for the error in the primal variable $u$. THEOREM 3.8 ( $L_{2}$-convergence) Assuming that $\Omega$ is a convex domain, we have

$$
\left\|u-u^{h}\right\|_{L_{2}(\Omega)} \leqslant C h^{2}\left(|u|_{H^{2}(\Omega)}+|\lambda|_{H^{1 / 2}\left(\cup \Gamma_{j}\right)}\right) .
$$

Proof. Consider the dual problem of solving

$$
-\Delta z=u-u^{h} \text { in } \Omega, \quad z=0 \text { on } \partial \Omega .
$$

Defining $\partial_{n} z:=\boldsymbol{n}_{1} \cdot \nabla z$, we have, using Lemma 2.2 with $v^{h}=\pi_{h} z$ and $\mu^{h}=P_{l} \partial_{n} z$, that

$$
\begin{aligned}
\left\|u-u^{h}\right\|_{L_{2}(\Omega)}^{2}= & \sum_{i} \int_{\Omega_{i}} \nabla\left(u_{i}-u_{i}^{h}\right) \cdot \nabla\left(z-\pi_{h} z\right) \mathrm{d} x+\int_{\Gamma}\left(P_{l} \partial_{n} z-\partial_{n} z\right) \llbracket u-u^{h} \rrbracket \mathrm{d} s \\
& -\int_{\Gamma}\left(\lambda-\lambda^{h}\right) \llbracket \pi_{h} z \rrbracket \mathrm{d} s+j\left(\lambda^{h}, P_{l} \partial_{n} z\right) .
\end{aligned}
$$

Estimating each term on the right-hand side separately, we have, denoting the maximum second derivative of $z$ by $D^{2} z$ and using standard interpolation estimates, that

$$
\sum_{i} \int_{\Omega_{i}} \nabla\left(u_{i}-u_{i}^{h}\right) \cdot \nabla\left(z-\pi_{h} z\right) \mathrm{d} x \leqslant\left\|\nabla\left(u-u^{h}\right)\right\|_{0, h}\left\|\nabla\left(z-\pi_{h} z\right)\right\|_{L_{2}(\Omega)} \leqslant C h\left\|\nabla\left(u-u^{h}\right)\right\|_{0, h}\left\|D^{2} z\right\|_{L_{2}(\Omega)}
$$

and

$$
\begin{aligned}
\int_{\Gamma}\left(P_{l} \partial_{n} z-\partial_{n} z\right) \llbracket u-u^{h} \rrbracket \mathrm{d} s & \leqslant\left\|h_{\Gamma}^{1 / 2}\left(P_{l} \partial_{n} z-\partial_{n} z\right)\right\|_{L_{2}(\Gamma)}\left\|h_{\Gamma}^{-1 / 2} \llbracket u-u^{h} \rrbracket\right\|_{L_{2}(\Gamma)} \\
& \leqslant C h\left\|\llbracket u-u^{h} \rrbracket\right\|_{\frac{1}{2}, h, \Gamma}\left\|D^{2} z\right\|_{L_{2}(\Omega)} .
\end{aligned}
$$

We now note that

$$
\begin{aligned}
\left\|\llbracket u-u^{h} \rrbracket\right\|_{\frac{1}{2}, h, \Gamma} & =\left\|\llbracket u^{h} \rrbracket\right\|_{\frac{1}{2}, h, \Gamma} \leqslant C\left\|\llbracket u^{h} \rrbracket\right\|_{\frac{1}{2}, \Gamma}=C\left\|\llbracket u-u^{h} \rrbracket\right\|_{\frac{1}{2}, \Gamma} \\
& \leqslant C\left(\left\|u_{1}-u_{1}^{h}\right\|_{\frac{1}{2}, \Gamma}+\left\|u_{2}-u_{2}^{h}\right\|_{\frac{1}{2}, \Gamma}\right) \leqslant C\left\|\left(u-u^{h}, 0\right)\right\|_{0}
\end{aligned}
$$

by a trace inequality together with Poincaré's inequality, and we conclude that

$$
\int_{\Gamma}\left(P_{l} \partial_{n} z-\partial_{n} z\right) \llbracket u-u^{h} \rrbracket \mathrm{d} s \leqslant C h\left\|\left(u-u^{h}, 0\right)\right\|\left\|_{0}\right\| D^{2} z \|_{L_{2}(\Omega)} .
$$


Further, $\llbracket \pi_{h} z \rrbracket=\pi_{h}^{1} z-z+z-\pi_{h}^{2} z$ and

$$
\begin{aligned}
\pm \int_{\Gamma}\left(\lambda-\lambda^{h}\right)\left(\pi_{h}^{i} z-z\right) \mathrm{d} s & \leqslant C\left\|\lambda-\lambda^{h}\right\|_{-\frac{1}{2}, h, \Gamma}\left\|h^{-1 / 2}\left(\pi_{h}^{i} z-z\right)\right\|_{L_{2}(\Gamma)} \\
& \leqslant C h\left\|\lambda-\lambda^{h}\right\|_{-\frac{1}{2}, h, \Gamma}\left\|D^{2} z\right\|_{L_{2}(\Omega)}
\end{aligned}
$$

and finally, using the same argument as in the proof of Theorem 3.7, we have

$$
\begin{aligned}
j\left(\lambda^{h}, P_{l} \partial_{n} z\right) & \leqslant j\left(\lambda^{h}, \lambda^{h}\right)^{1 / 2} j\left(P_{l} \partial_{n} z, P_{l} \partial_{n} z\right)^{1 / 2} \\
& \leqslant C h j\left(\lambda^{h}, \lambda^{h}\right)^{1 / 2}\left\|D^{2} z\right\|_{L_{2}(\Omega)} .
\end{aligned}
$$

If $\Omega$ is convex then we have from (3.22) that $\left\|D^{2} z\right\|_{L_{2}(\Omega)} \leqslant C\left\|u-u^{h}\right\|_{L_{2}(\Omega)}$, and the result follows from Theorem 3.7.

\section{Numerical examples}

\subsection{Locking}

A typical example of the effect of too many Lagrange multipliers on the interface is given in Fig. 3. The domain is $\Omega=(0,3) \times(0,3)$ with $\Omega_{2}=(1,2) \times(1.5,2.5)$. We show the result obtained for a problem with a smooth solution using 3000 linear multipliers on each straight segment dividing $\Omega_{1}$ and $\Omega_{2}$, as well as the effect of edge stabilization with $\gamma=200$. The severe locking, due to overconstraining (failure to meet the inf-sup condition), is completely alleviated.

\subsection{Convergence}

On the same domain as in Section 4.1 we give the convergence of $u^{h}$ in the broken energy norm and $\lambda^{h}$ in the discrete half-norm $\|\cdot\|_{-\frac{1}{2}, h, \Gamma}$. In Fig. 4 we give an elevation of the approximate solution on the last mesh in a sequence. Here the exact solution is given by $u=(3-x) x(3-y) y$.

In Figs 5 and 6 we give convergence plots for the piecewise-constant and the piecewise-linear, continuous approximations of the multiplier. Twenty-two multipliers were used in the first mesh, and the
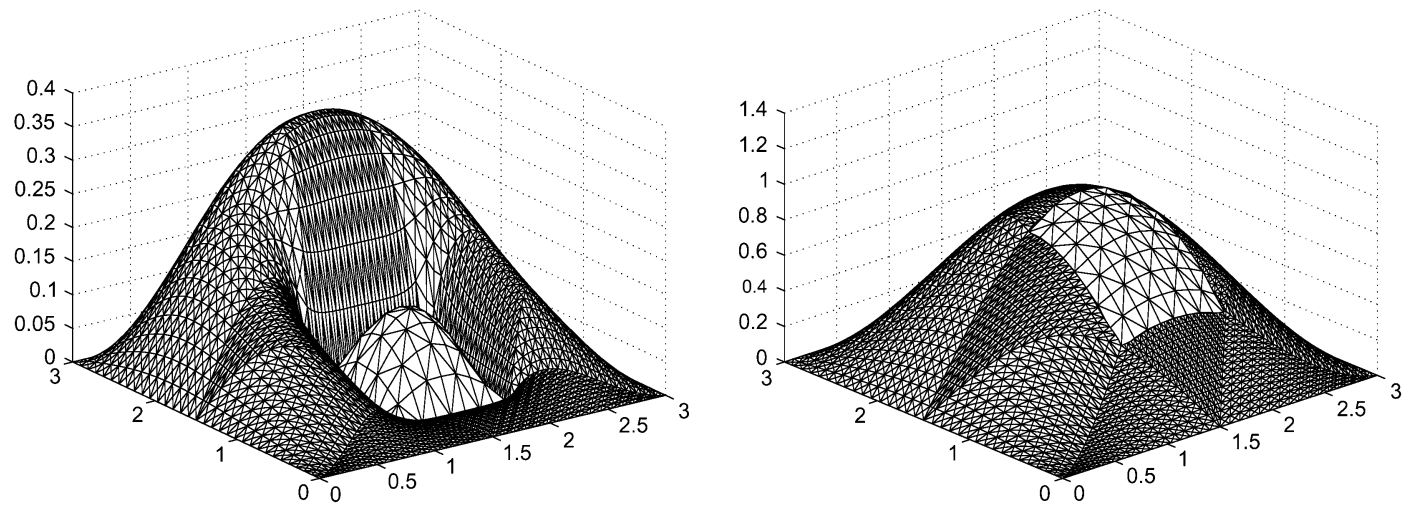

FIG. 3. Locking effect from inf-sup instability and the effect of stabilization. 


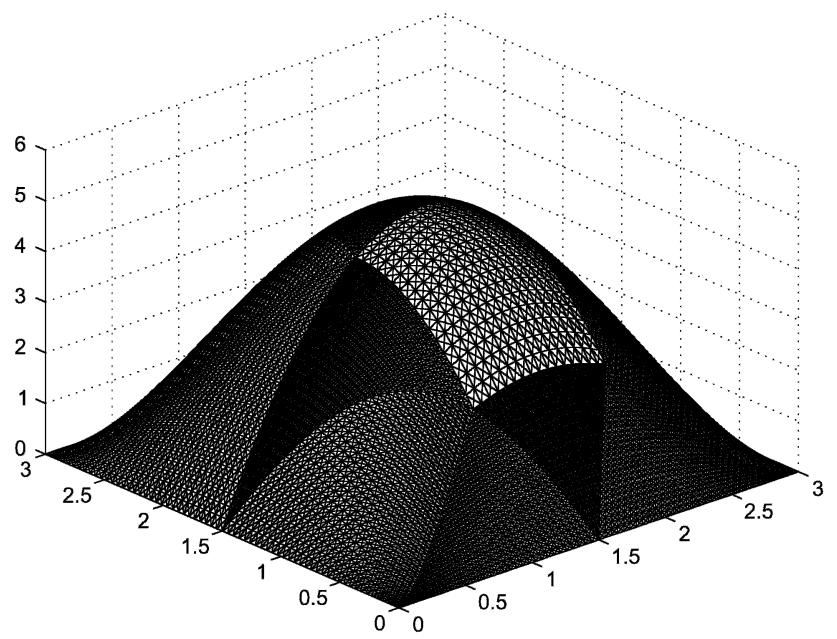

FIG. 4. Elevation of the exact solution on the last mesh in a sequence.

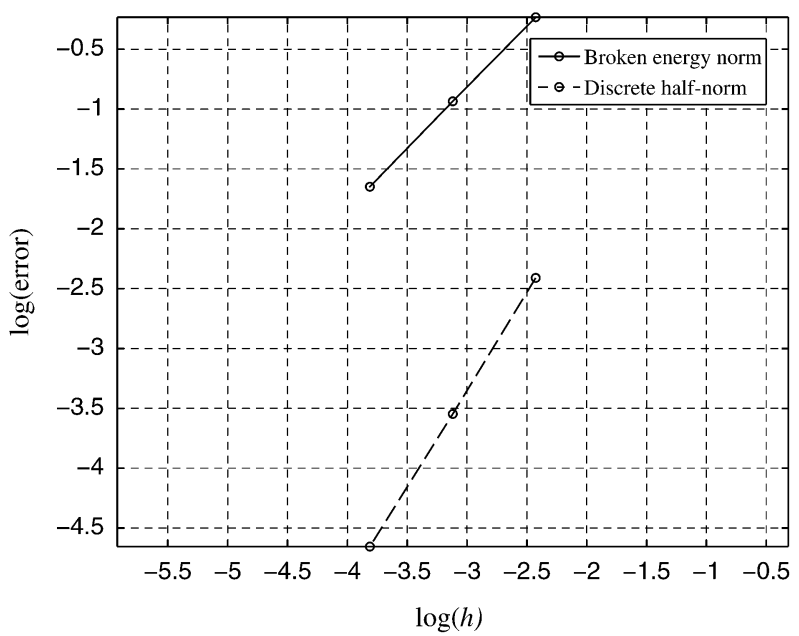

FIG. 5. Convergence obtained with piecewise-constant multipliers.

number was doubled in each successive mesh refinement. We set $\gamma=200$ in both cases. We note that the piecewise-constant multiplier gives a convergence (of approximately $\mathcal{O}\left(h^{3 / 2}\right)$ ) that is better than that of the linear multipliers (approximately $\mathcal{O}(h)$ ), which may be due to the fact that the normal derivative from $u^{h}$ indeed piecewise is constant. We remark that the convergence curves for $\lambda^{h}$ are given with respect to the mesh size for $u^{h}$. Since they are tied, this is of no consequence.

Finally, in Fig. 7 we give the convergence of $u^{h}$ in $L_{2}(\Omega)$, which is of second order, in agreement with (3.21). 


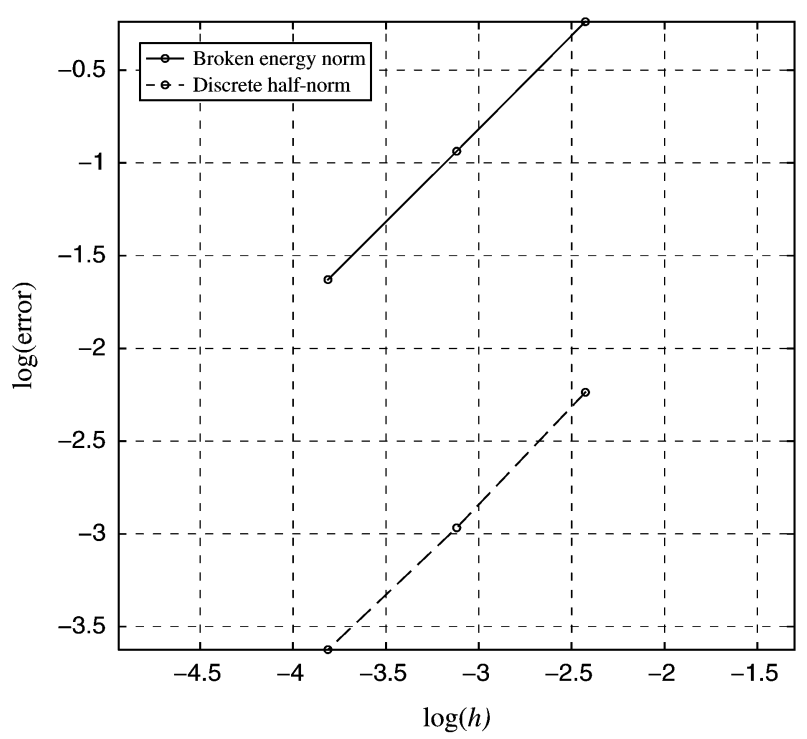

FIG. 6. Convergence obtained with piecewise-linear multipliers.

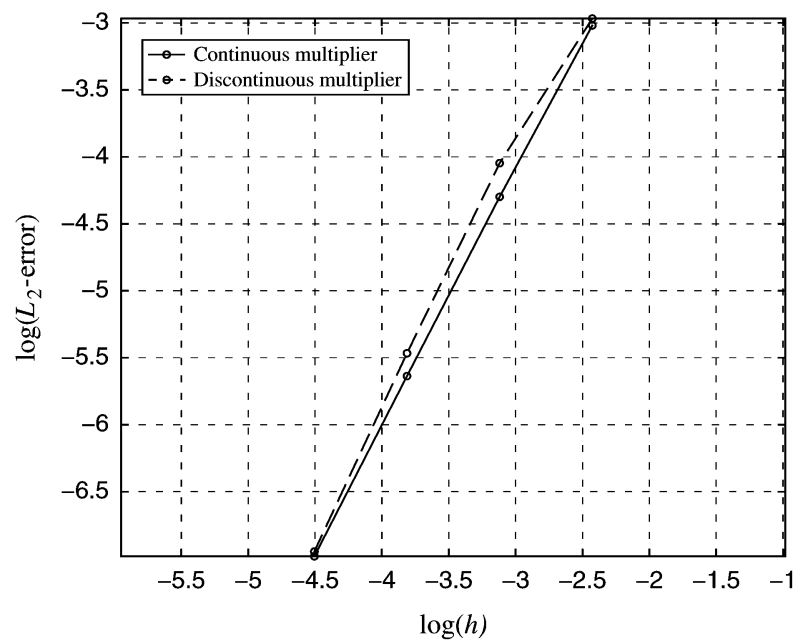

FIG. 7. Convergence of $\left\|u-u^{h}\right\|_{L_{2}(\Omega)}$ obtained with continuous and discontinuous multipliers.

\section{Concluding remarks}

We have proposed a weakly consistent interior penalty stabilization of the Lagrange multipliers in the numerical solution of elliptic interface problems. Unlike other stabilization schemes, the stabilization does not directly couple the discretizations of the primal solution and the multiplier. In our numerical experience the choice of the stabilization parameter does not much affect the primal solution, though oscillations in the approximation of the multiplier may occur if it is chosen too small. Since the multiplier 
can alternatively be derived from the primal solution, this may be of little consequence in practice. We strongly believe that, in particular, for the piecewise-constant approximation our scheme offers a good alternative to stable multiplier methods such as the mortar method, as well as to alternative stabilization methods.

\section{REFERENCES}

Barbosa, H. J. C. \& Hughes, T. J. R. (1992) Boundary Lagrange multipliers in finite-element methods-error analysis in natural norms. Numer. Math., 62, 1-15.

Becker, R., Hansbo, P. \& Stenberg, R. (2003) A finite element method for domain decomposition with non-matching grids. ESAIM: Math. Model. Numer. Anal., 37, 209-225.

Brezzi, F. \& Fortin, M. (1991) Mixed and Hybrid Finite Element Methods. New York: Springer.

Burman, E. \& HANSBO, P. (2004) Edge stabilization for Galerkin approximations of convection-diffusion problems. Comput. Methods Appl. Mech. Eng., 193, 1437-1453.

Hansbo, P., Lovadina, C., Perugia, I. \& Sangalli, G. (2005) A Lagrange multiplier method for the finite element solution of elliptic interface problems using non-matching meshes. Numer. Math., 100, 91-115.

Lions, J. L. \& Magenes, E. (1968) Problèmes aux limites non homogènes et applications, vol. 1. Paris: Dunod. Thомée, V. (1997) Galerkin Finite Element Methods for Parabolic Problems. Berlin: Springer.

Wohlmuth, B. I. (2001) Discretization Methods and Iterative Solvers Based on Domain Decomposition. Berlin: Springer. 